\title{
Myocardial Tissue Characterization: Histological and Pathophysiological Correlation
}

\author{
T. A. Treibel • S. K. White • J. C. Moon
}

Published online: 15 January 2014

(C) Springer Science+Business Media New York 2014

\begin{abstract}
Cardiovascular magnetic resonance imaging (CMR) has become the gold standard not only for cardiac volume and function quantification, but for a key unique strength: non-invasive myocardial tissue characterization. Several different techniques, separately or in combination, can detect and quantify early and established myocardial pathological processes permitting better diagnosis, prognostication and tracking of therapy. The authors will focus on the histological and pathophysiological evidence of these imaging parameters in the characterization of edema, infarction, scar and fibrosis. In addition to laying out the strengths and weaknesses of each modality, the reader will be introduced to rapid developments in T1 and T2 mapping as well as the use of contrast-derived extracellular volume for quantification of diffuse fibrosis.
\end{abstract}

Keywords Myocardial fibrosis · Edema $\cdot \mathrm{T} 1 \cdot \mathrm{T} 2 \cdot \mathrm{LGE} \cdot$ Late enhancement

\section{Introduction}

Tissue characterization and measurement of fibrosis is a mainstay of clinical care in respiratory medicine, nephrology and hepatology, but in cardiology this has been limited to the few patients who received cardiac biopsies. The emergence of

This article is part of the Topical Collection on Cardiac Magnetic Resonance

T. A. Treibel $\cdot$ S. K. White J. C. Moon

Department of Cardiology, The Heart Hospital, University College

London Hospitals NHS Trust, London, UK

J. C. Moon $(\triangle)$

The Heart Hospital Imaging Centre, University College London

Hospitals NHS Trust, 16-18 Westmoreland Street, London W1G

$8 \mathrm{PH}, \mathrm{UK}$

e-mail: j.moon@ucl.ac.uk cardiovascular magnetic resonance (CMR) is changing this. Myocardial fibrosis is inherently an important clinical parameter because fibrosis represents one of the hallmarks of pathological remodeling of the myocardium [1-4]. CMR has established itself over the last decade not only as the gold standard for cardiac chamber volume and function quantification, but also for non-invasive myocardial tissue characterization. Its strength lies in the use of multiple parameters to characterize myocardium. The development of imaging parameters for the quantification of edema, infarction and scar has been followed by their adoption by the CMR community for non-invasive tissue characterization, in particular in acute and chronic myocardial infarction (MI). During an acute MI, occlusion of a coronary artery territory leads to myocyte necrosis, which spreads from the subendocardial to the subepicardial layers (the 'wavefront phenomenon'). Without reperfusion, or collateral blood supply, complete necrosis of the perfusion territory distal to the occluded coronary artery ensues. The total amount of myocardium at risk of necrosis is termed the 'area-atrisk' (AAR). In general the resulting irreversibly injured myocardium (i.e., chronic myocardial infarct) is smaller than the AAR; furthermore, early reopening of the infarct-related artery can interrupt this process and lead to salvage of myocardium.

This review will focus on the histological and pathophysiological evidence of these imaging parameters in the characterization of infarction, scar and fibrosis across myocardial pathologies. The majority of animal models are based on myocardial ischemia and infarction, therefore large amounts of histological and pathophysiological evidence arises from the exemplar conditions of acute and chronic myocardial infarction.

\section{Fundamental Properties of Tissues}

Magnetic resonance characteristics of protons vary between tissues, depending on the configuration of atoms in the tissues. 
These inherent differences can be exploited to generate differing signals from separate tissues: T1 relaxation time (longitudinal relaxation time) is determined by how rapidly protons re-equilibrate their spins with their environment following a radiofrequency $(\mathrm{RF})$ pulse. The native (non-contrast) myocardial T1 varies with water content and increases in cases of edema, fibrosis or infiltration of the extracellular space (e.g., in cardiac amyloid). Inherently, it embodies composite signal from both cells and interstitium, and varies with measurement technique and MRI field strength. Regional difference in T1 can be visualized by T1-weighted MR sequences or directly estimated by T1 mapping. T2 relaxation time (spin-spin relaxation time) is determined by how rapidly the refocused transverse signal decays after a RF pulse (in contrast to the rate of spin dephasing, which is called T2*). T2 is shorter when water is tightly bound to large molecules like collagen, but longer when water is free. T2-weighted images emphasize tissues with long $\mathrm{T} 2$ or in conditions like where myocardial edema is present; new T2 mapping sequences are able to quantify $\mathrm{T} 2$ directly.

\section{Exploring Pathophysiology with T1 and T2 Imaging: Acute Infarction}

Technical advances in $\mathrm{T} 1$ and $\mathrm{T} 2$-weighted imaging have allowed in-vivo visualization and accurate quantification of myocardial edema, a substantial feature of myocardial infarction (MI) with ischemic and reperfusion injury. Preliminary work by Higgins et al. in the 1980s in a dog infarct model showed $\mathrm{T} 1$ and $\mathrm{T} 2$ elevation in infarction [5]. The observed changes were theoretically consistent with myocardial edema and correlated with the measurements of myocardial water content estimated by wet-weight to dry-weight ratios. This early work aimed to develop non-contrast methods for diagnosing MI but overestimated infarct size. It was not until a decade later that $\mathrm{T} 2$-weighted images were clearly shown to correspond to the area at risk (AAR) during an acute MI rather than the eventual chronic infarct size.

In 1993, Garcia-Dorado et al. were the first to show, in an ex-vivo dog model of both non-reperfused and reperfused infarction, that $\mathrm{T} 2$-weighted images represent the AAR and to confirm a tight relationship between myocardial $\mathrm{T} 2$ and tissue water content [6]. Aletras et al. then showed that in-vivo T2-weighted images enabled measurement of the area at risk and compared well with fluorescent microspheres [7]. Tilak et al. reported that $\mathrm{T} 2$-weighted images correspond to the area at risk in a canine model of non-reperfused infarcts, using first-pass perfusion during coronary occlusion as reference [8]. More recently, Ugander et al. [9] validated T2-weighted and pre-contrast T1-weighted images as measures of the AAR against whole-heart microsphere reference standards.
In humans, following initial work noting that T2-weighted images could be used to differentiate acute from chronic myocardial infarction [10], Berry et al. [11] showed that T2weighted images in acute myocardial infarctions were transmural and had hyperintense regions in the distribution of the culprit coronary artery. Friedrich et al. recognized that T2-weighted images could be used to image salvaged myocardium in humans [12]. Carlsson et al. [13] used sestamibi (injected prior to acute percutaneous coronary intervention and imaged with SPECT) to validate T2-weighted images with regard to determination of area at risk and found excellent correlation, and showed that the AAR was similar on days 1-7 post MI but then decreased over 6 months. This fact alone cements the use of CMR in clinical studies and was recently confirmed by work at 3 Tesla by Dall'Armellina and colleagues [14]. The use of T2-weighted CMR has not been restricted to acute infarction, but has also established itself in the diagnostic pathway for investigation and characterization of myocardial inflammation, infiltration and masses. This follows a pattern of myocardial tissue characterization being used and developed in infarction where large differences exist between normal and abnormal tissue and dissemination into other focal diseases, particularly scar in non-ischemic cardiomyopathies before use for diffuse myocardial abnormalities. In a process of incremental value rather than succession, technical development has followed a similar pathway with T1/T2-weighted imaging followed by late gadolinium enhancement technique, and, more recently, quantitative $\mathrm{T} 1$ and $\mathrm{T} 2$ mapping techniques. These will be discussed subsequently.

\section{Conventional Scar Imaging by Late Gadolinium Enhancement}

Historically, myocardial scar has been visualized and measured directly on histological sections using stains specific for the connective tissue in the extracellular space [1]. However, to allow non-invasive measurement, tracers were needed that are confined to the extracellular space. These tracers needed to distribute homogeneously through the extracellular space but not enter cells; be highly water but not fat soluble; not be adsorbed, actively transported, protein-bound or metabolized; be non-toxic, stable and be cleared freely from the body; and finally be easily measured. Gd-DTPA fulfills these requirements as a tracer; it diffuses rapidly from the vascular space into most extracellular tissue fluid, but not to the intracellular space, leading to the term 'extracellular contrast agent'. Gadolinium is a paramagnetic metal and because it has the most unpaired electrons [15], is the most efficient $\mathrm{T} 1$ relaxing agent, and also shortens $\mathrm{T} 2$ and $\mathrm{T} 2 *$. In the presence of gadolinium, $\mathrm{T} 1$ of tissues is potently shortened, resulting in increased signal on T1-weighted images and appearing bright on a $\mathrm{T} 1$ inversion recovery image. The relaxation rate (R1 or $1 / \mathrm{T} 1)$ is directly 
proportional to the concentration of gadolinium. Gadoliniumbased contrast agents are safe, with serious adverse events being extremely rare with an incidence less than 1 in 100,000 . Its safety profile and effect on the fundamental properties of T1 make it an ideal MR contrast agent.

\section{Pathophysiological Basis of Gadolinium Contrast Imaging}

To understand the pathophysiological basis of gadolinium contrast imaging, the pharmacokinetics of Gd-DTPA need to be considered. Following an intravenous bolus, gadolinium enters the myocardium down a concentration gradient ("wash-in phase"), and later, as the gadolinium has been cleared from the blood pool, it returns to the blood pool down the reversed concentration gradient ("wash-out phase"). This occurs over seconds to minutes in healthy myocardium, where cells are tightly packed and cell membranes are intact. In acutely infarcted tissue, these kinetic effects are delayed due to changes in coronary flow rates, capillary permeability, and functional capillary density [16]. Furthermore, the volume of distribution is larger due to ruptured cell membranes allowing Gd-DTPA to passively diffuse into the cellular compartment [17]. Combined, Gd-DTPA accumulates and lingers in the infarcted tissue giving shorter T1s. In chronic infarct (i.e., established scar), the volume of distribution is larger because of a significant reduction in living myocytes, which are replaced by a dense, hydrated collagen matrix, resulting in a higher accumulation of Gd-DTPA.

In practical terms, following administration of a bolus of Gd-DTPA, an inversion recovery sequence is performed, with the inversion time (TI) set manually by the operator. The TI is set in order to null "normal" remote myocardium which then appears black, resulting in the greatest image intensity difference between normal and infarcted tissue [18]. This late gadolinium enhancement (LGE) enables direct quantification of the spatial extent of focal scar, but the ability for absolute quantification of either diffuse, background fibrosis or density of focal scar is lost. Despite the extremely high resolution and correlation between histology and LGE in ex-vivo rat hearts at high field strength [19••], it remains unknown what the clinical minimum "critical mass" of collagen is for LGE detection by. LGE imaging and analysis relies on thresholding techniques for quantification. Depending on the disease and technique, there can be a two-fold difference [20]. New T1 mapping sequences, which will be discussed later, allow objective quantification of signal magnitude, which is potentially directly comparable between studies and patients (Fig. 1).

\section{Histological Basis of Scar Imaging by CMR}

Initial animal work in the 1980s was performed with manganese chloride [21] and later gadolinium chelates in canine models of myocardial infarction showing differential and time-varying effects on relaxation times of normal and infarcted myocardium $[22,23]$. The development of inversion recovery late gadolinium enhancement (LGE) MRI [24] and extensive preclinical and clinical validation studies established MRI as a reference standard method for imaging myocardial infarction and viability. Kim et al. published a seminal canine infarct study that documented that gadolinium-enhanced MRI depicted infarcted myocardium with remarkable fidelity [25]. That work was supported by extensive validations of gadolinium concentrations in acutely infarcted myocardium [26]. Careful studies resolved infarct from the ARR and remote myocardium to determine that gadolinium enhances acute and chronic MI [27]. The histological validation of LGE has been taken close to a cellular level by Schelbert et al., who performed extremely high resolution ex-vivo LGE imaging on rats at 7 Tesla and compared with histological sections showing a remarkable correlation $\left(\mathrm{R}^{2}=0.96 ; \mathrm{p}<0.001\right.$ - see Fig. 2) [19••].

Clinical validation studies established that gadolinium differentiated viable myocardium from infarcted myocardium in patients. Kim et al. showed that the transmural extent of infarction predicted the recovery of contractile function after revascularization [28], a study confirmed by Selvanayagam et al. [29] - provided revascularization did not induce infarction. LGE-CMR has now become the gold standard for noninvasive quantification of focal fibrosis, in particular infarct size, with the spatial resolution needed to determine the transmural extent of infarction. The burden of LGE predicts function recovery after MI [30] and revascularization [29], as well as mortality and MACE [31-33]. Single-photon emission tomography (SPECT) and positron-emission tomography (PET) validation studies confirmed that CMR LGE had equal or better sensitivity for infarction [34], in particular in small subendocardial infarctions seen on histology [35].

The LGE method has proved to be reproducible [36], and was robust enough to perform with high sensitivity and specificity in a multicenter clinical trial [37]. LGE has therefore established itself as the gold standard method for assessment of scar in both ischemic and non ischemic heart diseases including cardiomyopathy [38], myocarditis [39], aortic stenosis induced pressure-overload hypertrophy [40] and infiltrative diseases [41].

\section{Advanced Scar Imaging and ECV}

The extracellular matrix constitutes around $6 \%$ of the normal heart and serves to anchor cardiac muscle cells, thus determining cardiac structure and function [2]. As described, the LGE technique is the gold standard for the assessment of focal fibrosis $[42,43]$. However, LGE is not able to quantify diffuse myocardial fibrosis, which causes non-focal expansion of the 
Fig. 1 Multi-parametric imaging in acute myocardial infarction. Basal short axis slice in a patient scanned 3 days after acute inferior myocardial infarction. Native (ShMOLLI [54•]) T1 maps (a) and T2 maps (b) show elevated values in the area-at-risk (myocardium between double lines); an ECV map (c) shows the highest ECV values

corresponding to the area of late gadolinium enhancement (d) or acute infarction. Intra-myocardial hemorrhage can be identified by low $\mathrm{T} 2$ values in the core of the risk zone (thin black arrow). A small area of microvascular obstruction in (d) does not allow contrast to enter and appears as a core of low ECV values in (c) (thin white arrows)
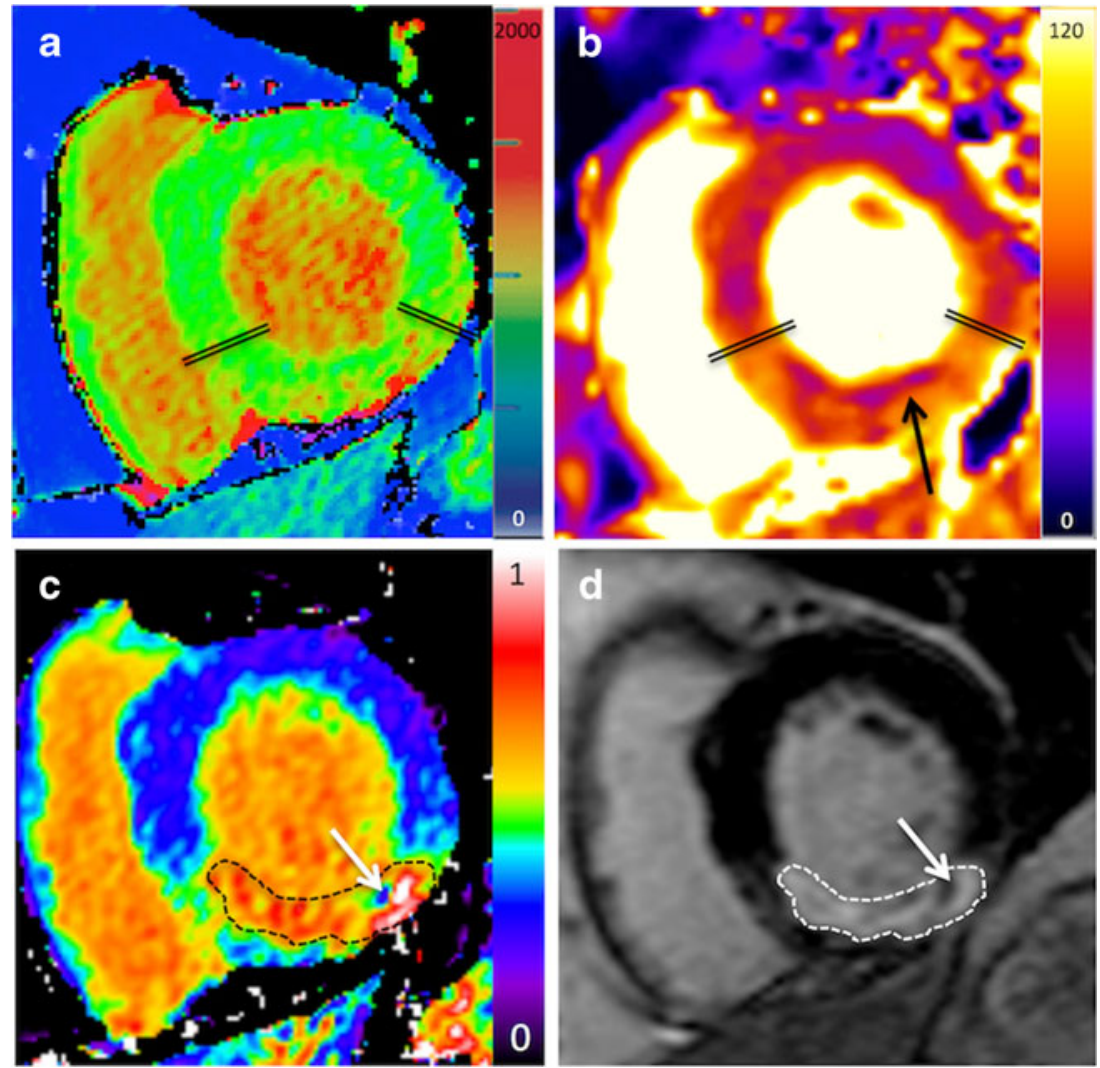

extracellular space. The current gold standard test for quantifiying fibrosis is myocardial biopsy, but this is associated with the potential for significant morbidity and mortality and is also prone to sampling error [44]. Surrogate tests lack sensitivity and/or specificity. New techniques for the quantification of the extracellular space have therefore been developed.

Early in-vitro CMR work by Kehr et al. on human myocardium obtained postmortem compared T1 values, calculated
Fig. 2 Histology and late gadolinium enhancement images in a rat model. Late gadolinium enhancement (LGE) tracks fibrosis to nearly the cellular level in this post infarction ex-vivo rat heart. LGE images were taken ex-vivo at 7 Tesla with a 3D gradient echo sequence (a and $\mathbf{b}$ ) were compared with histological sections stained with Masson trichrome (c-e). Adapted from Schelbert et al. Circulation CVI 2010 [19••]

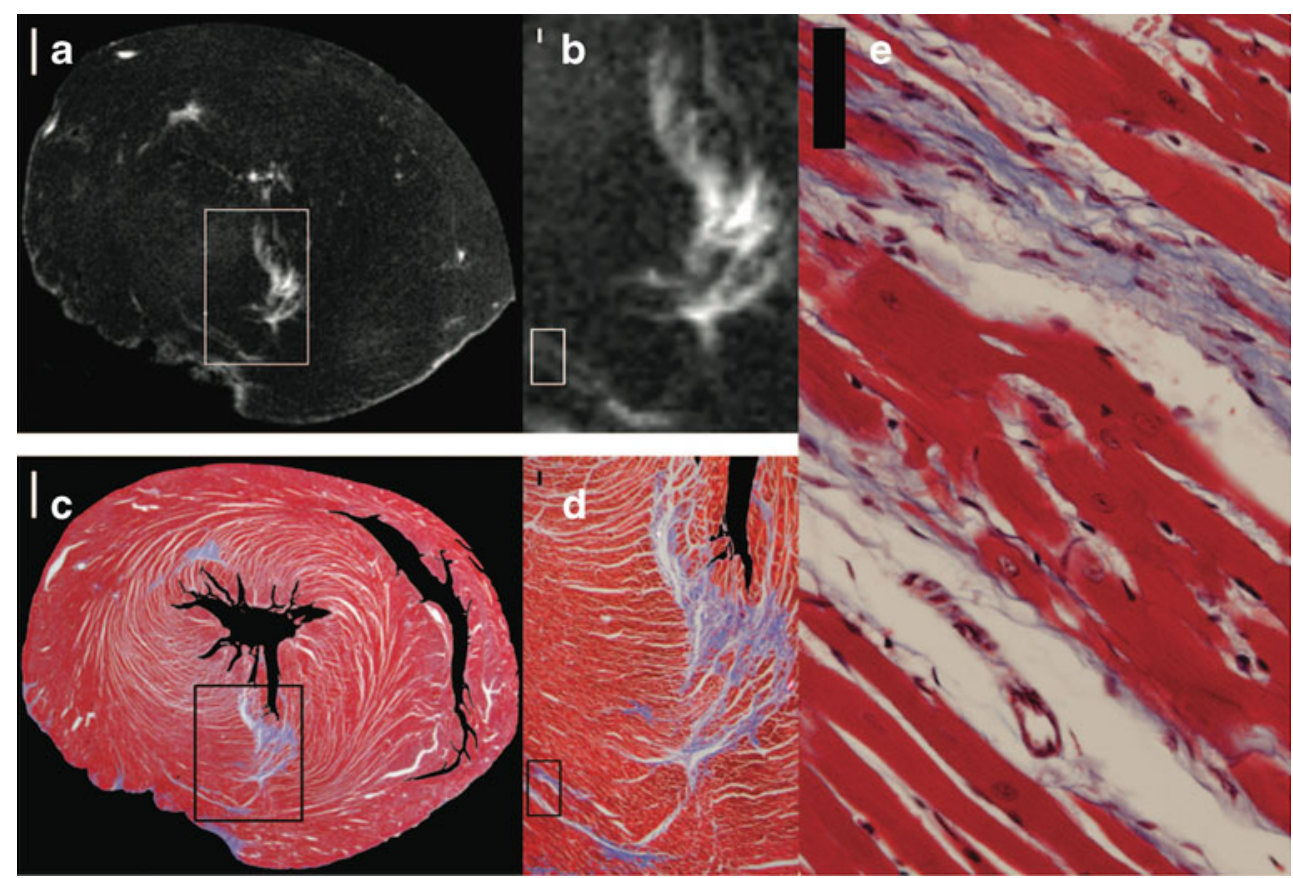


from the inversion recovery signal curves, with collagen volume fraction, determined by the picrosirius red method, and showed a significant correlation between the two methods [45]. Flett et al. then developed an extracellular volume technique and validated it in patients with severe aortic stenosis and hypertrophic cardiomyopathy [46••]: by calculating preand post-contrast $\mathrm{T} 1$ values from inversion recovery signal curves, the extracellular volume fraction (ECV) was derived by incorporating the blood volume of distribution (1-hematocrit). The ECV showed a high correlation with the collagen volume fraction of biopsies obtained intraoperatively in this cohort. This work has been replicated in transplant hearts, shorter protocols and with newer, faster T1 mapping sequences (see Fig. 3) [47, 48]. After administration of gadolinium, $\mathrm{T} 1$ is dominated by and inversely proportional to the amount of gadolinium present in a tissue. Measuring $\mathrm{T} 1$ after contrast provides a value linked to the interstitium and has been applied to patients with heart failure [49], but post-contrast $\mathrm{T} 1$ also varies with gadolinium dose, time post bolus, and importantly patient specific factors such as heart rate, clearance rate, body composition, and hematocrit. If the change in $\mathrm{T} 1$ pre- and post-contrast is measured in both blood and myocardium after equilibration of the contrast distribution, the partition coefficient can be calculated. By correcting for the hematocrit, the myocardial extracellular volume (ECV) is derived - a more stable and biologically significant biomarker. ECV divides the myocardium into two compartments (extracellular and cellular), and allows therefore noninvasive quantification of the myocardial interstitial volume and its reciprocal, cell volume; there is even some evidence that the contrast kinetics could be used to obtain cell sizedependent parameters [50]. The emerging evidence suggests that ECV measurement is the more robust parameter [51 • $]$ and the first of a new raft of papers on prognosis showing that $\mathrm{ECV}$, in unselected patients, predicts outcomes at least as strongly as left ventricular ejection fraction $[52 \bullet \bullet, 53]$.
Fig. 3 Histological validation of diffuse fibrosis in severe aortic stenosis. Top panel (a) showing correlation of extracellular volume (ECV) measured by CMR with histological collagen volume fraction in patients with severe aortic stenosis. The dashed lines represent $95 \%$ confidence intervals. Bottom row (b-d) showing histology samples of mild (b), moderate (c) and severe (d) diffuse myocardial fibrosis. Picrosirus red stains collagen (fibrosis) red/pink, and counter stains myocytes yellow. Collagen volume fraction (\%) was calculated by automated subtraction of these 2 color stains. Adapted from White et al. JACC CVI 2013 [47]

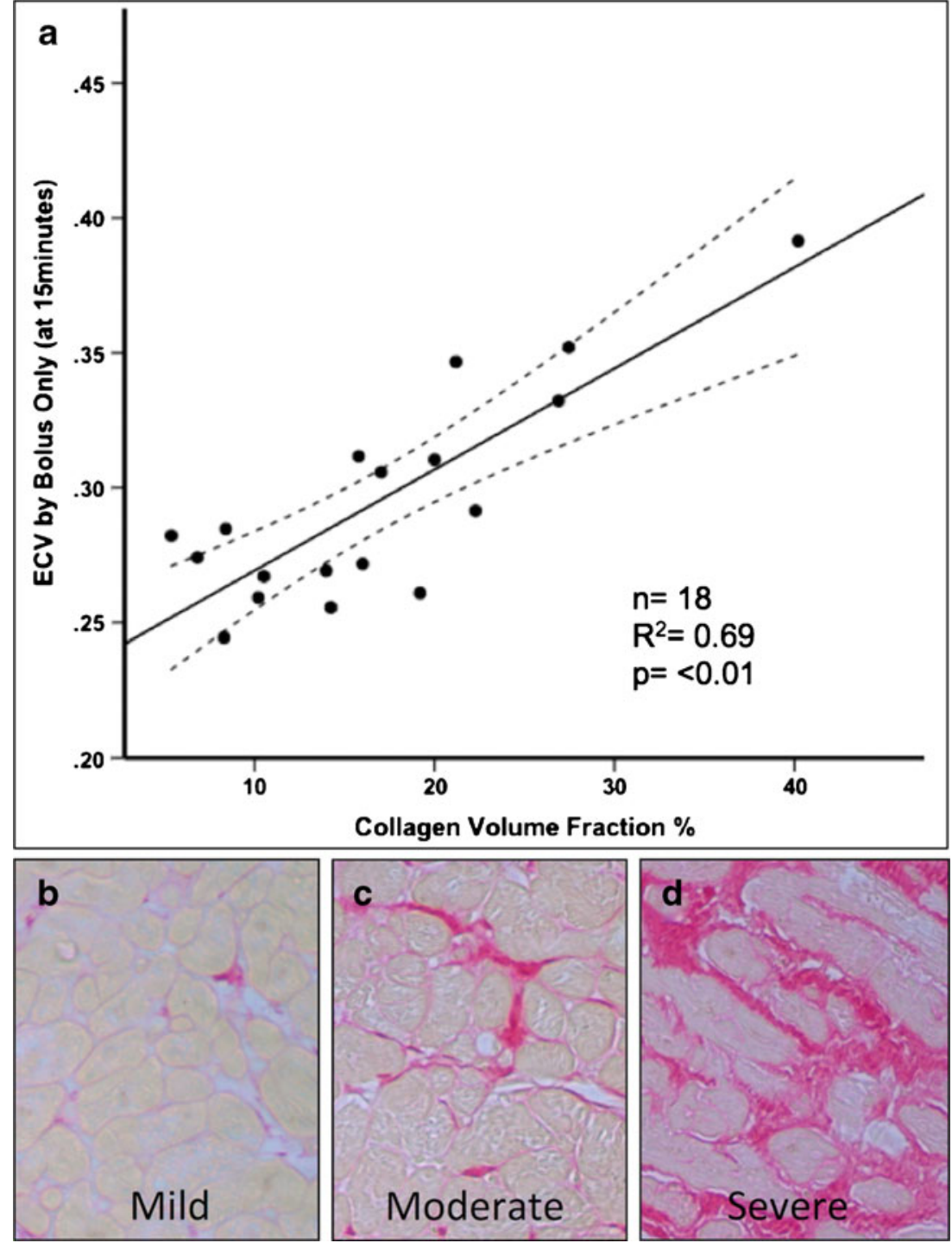




\section{From Signal Intensity to Direct Quantification - Pixel-by-Pixel Mapping}

The T1 mapping field is rapidly advancing, and advances have brought it close to being used in clinical practice: Single breath-hold mapping with MOLLI, ShMOLLI and SASHA sequences $[54 \bullet, 55 \bullet, 56 \bullet$ (where each pixel carries the $\mathrm{T} 1$ value) have replaced previous multi breath-hold methods. Newer refinements include shorter breath-holds, better sequence design, and lately, ECV maps with pixel-by-pixel representation of ECV for a simplified detection and quantitative display of focal and diffuse fibrosis (see Fig. 1) [57••]. There is an apparent spectrum of interstitial expansion with cardiac amyloid, aortic stenosis and cardiomyopathy having large changes in ECV; but age and gender have much smaller or possibly no contributions [58] once partial volume issues are accounted for. The ratio of pathophysiological signal to measurement error is a key determinant of test performance. T1 mapping appears especially robust in detecting disease characterized by high ECV, such as amyloid (where the ECV is often $>0.5$ ) [59], or marked native $\mathrm{T} 1$ contrast diseases such as Anderson-Fabry disease (where fat storage makes native T1 fall) [60]. Little biopsy histological correlation is available in these diseases however. Native T1 mapping has also been used for identification of diffuse myocardial fibrosis in aortic stenosis and non-ischaemic cardiomyopathies [61, 62], as well as the detection of acute myocarditis and showed excellent and superior diagnostic performance compared with T2-weighted CMR [63].

A key task will be to transition from an early development phase with competing methodologies, to more standardized methodologies sufficient for use to: diagnose disease; define mechanistic pathways of disease affecting the interstitium, the myocyte or both; change therapy; and employ ECV as a surrogate endpoint in trials of drug development. This is the aim underpinning the recently formed T1 mapping development group and the T1 mapping consensus statement [64••]. The consensus statement also highlights that we are currently using a simple model to explain far more complex in-vivo biological and molecular structure: the interaction between cardiac myocytes and the cardiac interstitium. Effects such as magnetization transfer, diffusion distance and time, contrast mechanisms, trans-cytolemmal water exchange rate, flow, $\mathrm{T} 2$ or $\mathrm{T} 2 *$ relaxation will require further investigation $[65,66]$.

\section{Other Techniques}

A new approach is trying to exploit the signal from collagenrelated water molecules, which have a very fast signal decay (short T2 and T2*) compared to water in soft tissue. Using ultra short echo time (UTE) MRI, this has been successfully applied to solid collagen-rich areas like ligaments and tendons. In a rat model of myocardial infarction, UTE was able to directly detect post-infarct fibrosis without the use of contrast agents [67]. Furthermore, myocardial tissue characterization with quantification of the extracellular space has also been performed with cardiac computed tomography - in dogs [68] and humans $[69,70]$ - and may be attractive, despite the radiation dose, as it theoretically allows whole heart ECV quantification, although currently the technique has high noise compared to CMR.

\section{Conclusion}

Using the fundamental $\mathrm{T} 1$ and $\mathrm{T} 2$ properties of myocardial tissue as well as the highly reproducible postcontrast LGE method, CMR has established itself as the gold standard for non-invasive myocardial tissue characterization. ECV diffuse fibrosis measurement may take this a step further. We can now dichotomize the myocardium into its cellular and interstitial components and this will advance understanding of disease mechanisms as well as their relative contribution to cardiac vulnerability. This is a critically important issue, especially in the setting of heart failure where drug development strategies have produced few positive results over the last decade [71]. CMR offers an array of techniques, which separately or in combination can detect and quantify early and established myocardial pathological processes permitting better diagnosis, prognostication and tracking of therapy. This should in turn optimize the choice and timing of interventions, and enhance drug development.

Acknowledgments T.A. Treibel is supported by a grant from the UK National Institute for Health Research (Doctoral Research Training Fellowship: DRF-2013-06-102). S.K. White is supported by a grant from the British Heart Foundation (Clinical Research Training Fellowship: FS/ 10/ 72/28568). J.C. Moon is supported by the Higher Education Funding Council for England. This work was undertaken at the University College London Hospital and University College London, which received a proportion of funding from the Department of Health's National Institute for Health Research Biomedical Research Centres funding scheme.

\section{Compliance with Ethics Guidelines}

Conflict of Interest Thomas A Treibel, Steven K White, and James C Moon declare that they have no conflict of interest.

Human and Animal Rights and Informed Consent This article does not contain any studies with human or animal subjects performed by any of the authors. 


\section{References}

Papers of particular interest, published recently, have been highlighted as:

- Of importance

•- Of major importance

1. Anderson KR, Sutton MG, Lie JT. Histopathological types of cardiac fibrosis in myocardial disease. J Pathol. 1979;128(2):7985. doi:10.1002/path.1711280205.

2. Rossi MA. Pathologic fibrosis and connective tissue matrix in left ventricular hypertrophy due to chronic arterial hypertension in humans. J Hypertens. 1998;16(7):1031-41.

3. Weber KT, Brilla CG. Myocardial fibrosis and the reninangiotensin-aldosterone system. J Cardiovasc Pharmacol. 1992;20 Suppl 1:S48-54.

4. Beltrami CA, Finato N, Rocco M, Feruglio GA, Puricelli C, Cigola $\mathrm{E}$, et al. Structural basis of end-stage failure in ischemic cardiomyopathy in humans. Circulation. 1994;89(1):151-63.

5. Higgins CB, Herfkens R, Lipton MJ, Sievers R, Sheldon P, Kaufman L, et al. Nuclear magnetic resonance imaging of acute myocardial infarction in dogs: alterations in magnetic relaxation times. Am J Cardiol. 1983;52(1):184-8.

6. Garcia-Dorado D, Oliveras J, Gili J, Sanz E, Perez-Villa F, Barrabes $\mathrm{J}$, et al. Analysis of myocardial oedema by magnetic resonance imaging early after coronary artery occlusion with or without reperfusion. Cardiovasc Res. 1993;27(8):1462-9.

7. Aletras AH, Tilak GS, Natanzon A, Hsu LY, Gonzalez FM, Hoyt Jr $\mathrm{RF}$, et al. Retrospective determination of the area at risk for reperfused acute myocardial infarction with T2-weighted cardiac magnetic resonance imaging: histopathological and displacement encoding with stimulated echoes (DENSE) functional validations. Circulation. 2006;113(15):1865-70. doi:10.1161/ CIRCULATIONAHA.105.576025.

8. Tilak GS, Hsu LY, Hoyt Jr RF, Arai AE, Aletras AH. In vivo T2weighted magnetic resonance imaging can accurately determine the ischemic area at risk for 2-day-old nonreperfused myocardial infarction. Invest Radiol. 2008;43(1):7-15. doi:10.1097/RLI. 0b013e3181558822.

9. Ugander M, Bagi PS, Oki AJ, Chen B, Hsu LY, Aletras AH, et al. Myocardial edema as detected by pre-contrast T1 and T2 CMR delineates area at risk associated with acute myocardial infarction. JACC Cardiovasc Imaging. 2012;5(6):596-603. doi:10.1016/j. jemg.2012.01.016.

10. Abdel-Aty H, Zagrosek A, Schulz-Menger J, Taylor AJ, Messroghli D, Kumar A, et al. Delayed enhancement and T2weighted cardiovascular magnetic resonance imaging differentiate acute from chronic myocardial infarction. Circulation. 2004;109(20):2411-6. doi:10.1161/01.CIR.0000127428. 10985.C6

11. Berry C, Kellman P, Mancini C, Chen MY, Bandettini WP, Lowrey $\mathrm{T}$, et al. Magnetic resonance imaging delineates the ischemic area at risk and myocardial salvage in patients with acute myocardial infarction. Circ Cardiovasc Imaging. 2010;3(5):527-35. doi:10. 1161/CIRCIMAGING.109.900761.

12. Friedrich MG, Abdel-Aty H, Taylor A, Schulz-Menger J, Messroghli D, Dietz R. The salvaged area at risk in reperfused acute myocardial infarction as visualized by cardiovascular magnetic resonance. J Am Coll Cardiol. 2008;51(16):1581-7. doi:10. 1016/j.jacc.2008.01.019.

13. Carlsson M, Ubachs JF, Hedstrom E, Heiberg E, Jovinge S, Arheden H. Myocardium at risk after acute infarction in humans on cardiac magnetic resonance: quantitative assessment during follow-up and validation with single-photon emission computed tomography. JACC Cardiovasc Imaging. 2009;2(5):569-76. doi: 10.1016/j.jcmg.2008.11.018.

14. Dall'Armellina E, Karia N, Lindsay AC, Karamitsos TD, Ferreira V, Robson MD, et al. Dynamic changes of edema and late gadolinium enhancement after acute myocardial infarction and their relationship to functional recovery and salvage index. Circ Cardiovasc Imaging. 2011;4(3):228-36. doi:10.1161/CIRCIMAGING.111. 963421.

15. Nair V, Butany J. Heart transplant biopsies: interpretation and significance. J Clin Pathol. 2010;63(1):12-20. doi:10.1136/jcp. 2009.072462.

16. Kim RJ, Chen EL, Lima JA, Judd RM. Myocardial Gd-DTPA kinetics determine MRI contrast enhancement and reflect the extent and severity of myocardial injury after acute reperfused infarction. Circulation. 1996;94(12):3318-26.

17. Flacke SJ, Fischer SE, Lorenz CH. Measurement of the gadopentetate dimeglumine partition coefficient in human myocardium in vivo: normal distribution and elevation in acute and chronic infarction. Radiology. 2001;218(3):703-10.

18. Kim RJ, Shah DJ, Judd RM. How we perform delayed enhancement imaging. J Cardiovasc Magn Reson. 2003;5(3):505-14.

19.• Schelbert EB, Hsu LY, Anderson SA, Mohanty BD, Karim SM, Kellman $\mathrm{P}$, et al. Late gadolinium-enhancement cardiac magnetic resonance identifies postinfarction myocardial fibrosis and the border zone at the near cellular level in ex vivo rat heart. Circ Cardiovasc Imaging. 2010;3(6):743-52. doi:10.1161/ CIRCIMAGING.108.835793. The authors have taken histological validation of LGE close to a cellular level, performing extremely high-resolution ex-vivo LGE imaging on rats at 7 Tesla and compared with histological sections showing a remarkable correlation.

20. Flett AS, Hasleton J, Cook C, Hausenloy D, Quarta G, Ariti C, et al. Evaluation of techniques for the quantification of myocardial scar of differing etiology using cardiac magnetic resonance. JACC Cardiovasc Imaging. 2011;4(2):150-6. doi:10.1016/j.jcmg.2010. 11.015.

21. Goldman MR, Brady TJ, Pykett IL, Burt CT, Buonanno FS, Kistler JP, et al. Quantification of experimental myocardial infarction using nuclear magnetic resonance imaging and paramagnetic ion contrast enhancement in excised canine hearts. Circulation. 1982;66(5): 1012-6.

22. Wesbey GE, Higgins CB, McNamara MT, Engelstad BL, Lipton MJ, Sievers R, et al. Effect of gadolinium-DTPA on the magnetic relaxation times of normal and infarcted myocardium. Radiology. 1984;153(1):165-9.

23. Wesbey G, Higgins CB, Lanzer P, Botvinick E, Lipton MJ. Imaging and characterization of acute myocardial infarction in vivo by gated nuclear magnetic resonance. Circulation. 1984;69(1):125-30.

24. Simonetti OP, Kim RJ, Fieno DS, Hillenbrand HB, Wu E, Bundy $\mathrm{JM}$, et al. An improved MR imaging technique for the visualization of myocardial infarction. Radiology. 2001;218(1):215-23.

25. Kim RJ, Fieno DS, Parrish TB, Harris K, Chen EL, Simonetti O, et al. Relationship of MRI delayed contrast enhancement to irreversible injury, infarct age, and contractile function. Circulation. 1999;100(19): 1992-2002.

26. Rehwald WG, Fieno DS, Chen EL, Kim RJ, Judd RM. Myocardial magnetic resonance imaging contrast agent concentrations after reversible and irreversible ischemic injury. Circulation. 2002;105(2):224-9.

27. Fieno DS, Kim RJ, Chen EL, Lomasney JW, Klocke FJ, Judd RM. Contrast-enhanced magnetic resonance imaging of myocardium at risk: distinction between reversible and irreversible injury throughout infarct healing. J Am Coll Cardiol. 2000;36(6):1985-91.

28. Kim RJ, Wu E, Rafael A, Chen EL, Parker MA, Simonetti O, et al. The use of contrast-enhanced magnetic resonance imaging to identify reversible myocardial dysfunction. N Engl J Med. 2000;343(20):1445-53. doi:10.1056/NEJM200011163432003. 
29. Selvanayagam JB, Kardos A, Francis JM, Wiesmann F, Petersen SE, Taggart DP, et al. Value of delayed-enhancement cardiovascular magnetic resonance imaging in predicting myocardial viability after surgical revascularization. Circulation. 2004;110(12):1535-41. doi: 10.1161/01.CIR.0000142045.22628.74.

30. Choi KM, Kim RJ, Gubernikoff G, Vargas JD, Parker M, Judd RM. Transmural extent of acute myocardial infarction predicts long-term improvement in contractile function. Circulation. 2001;104(10): 1101-7.

31. Yan AT, Shayne AJ, Brown KA, Gupta SN, Chan CW, Luu TM, et al. Characterization of the peri-infarct zone by contrast-enhanced cardiac magnetic resonance imaging is a powerful predictor of postmyocardial infarction mortality. Circulation. 2006;114(1):32-9. doi:10.1161/CIRCULATIONAHA.106.613414.

32. Kwong RY, Chan AK, Brown KA, Chan CW, Reynolds HG, Tsang S, et al. Impact of unrecognized myocardial scar detected by cardiac magnetic resonance imaging on event-free survival in patients presenting with signs or symptoms of coronary artery disease. Circulation. 2006;113(23):2733-43. doi:10.1161/CIRCULATIONAHA.105. 570648.

33. Wu E, Ortiz JT, Tejedor P, Lee DC, Bucciarelli-Ducci C, Kansal P, et al. Infarct size by contrast enhanced cardiac magnetic resonance is a stronger predictor of outcomes than left ventricular ejection fraction or end-systolic volume index: prospective cohort study. Heart. 2008;94(6):730-6. doi:10.1136/hrt.2007.122622.

34. Klein C, Nekolla SG, Balbach T, Schnackenburg B, Nagel E, Fleck $\mathrm{E}$, et al. The influence of myocardial blood flow and volume of distribution on late Gd-DTPA kinetics in ischemic heart failure. J Magn Reson Imaging. 2004;20(4):588-93. doi:10.1002/jmri.20164.

35. Wagner A, Mahrholdt H, Holly TA, Elliott MD, Regenfus M, Parker M, et al. Contrast-enhanced MRI and routine single photon emission computed tomography (SPECT) perfusion imaging for detection of subendocardial myocardial infarcts: an imaging study. Lancet. 2003;361(9355):374-9. doi:10.1016/s0140-6736(03) 12389-6.

36. Mahrholdt H, Wagner A, Holly TA, Elliott MD, Bonow RO, Kim RJ, et al. Reproducibility of chronic infarct size measurement by contrast-enhanced magnetic resonance imaging. Circulation. 2002;106(18):2322-7.

37. Kim RJ, Albert TS, Wible JH, Elliott MD, Allen JC, Lee JC, et al. Performance of delayed-enhancement magnetic resonance imaging with gadoversetamide contrast for the detection and assessment of myocardial infarction: an international, multicenter, doubleblinded, randomized trial. Circulation. 2008;117(5):629-37. doi: 10.1161/CIRCULATIONAHA.107.723262.

38. Choudhury L, Mahrholdt H, Wagner A, Choi KM, Elliott MD, Klocke FJ, et al. Myocardial scarring in asymptomatic or mildly symptomatic patients with hypertrophic cardiomyopathy. J Am Coll Cardiol. 2002;40(12):2156-64.

39. Wagner A, Schulz-Menger J, Dietz R, Friedrich MG. Long-term follow-up of patients paragraph sign with acute myocarditis by magnetic paragraph sign resonance imaging. Magma. 2003;16(1): 17-20. doi:10.1007/s10334-003-0007-7.

40. Dweck MR, Joshi S, Murigu T, Alpendurada F, Jabbour A, Melina $\mathrm{G}$, et al. Midwall fibrosis is an independent predictor of mortality in patients with aortic stenosis. J Am Coll Cardiol. 2011;58(12):12719. doi:10.1016/j.jacc.2011.03.064.

41. Maceira AM, Prasad SK, Hawkins PN, Roughton M, Pennell DJ. Cardiovascular magnetic resonance and prognosis in cardiac amyloidosis. J Cardiovasc Magn Reson. 2008;10:54 doi:10.1186/1532429X-10-54.

42. Zemrak F, Petersen SE. Late gadolinium enhancement CMR predicts adverse cardiovascular outcomes and mortality in patients with coronary artery disease: systematic review and metaanalysis. Prog Cardiovasc Dis. 2011;54(3):215-29. doi:10.1016/j. pcad.2011.07.003.
43. Ismail TF, Prasad SK, Pennell DJ. Prognostic importance of late gadolinium enhancement cardiovascular magnetic resonance in cardiomyopathy. Heart. 2012;98(6):438-42. doi:10.1136/heartjnl2011-300814.

44. Becker AE, Heijmans CD, Essed CE. Chronic non-ischaemic congestive heart disease and endomyocardial biopsies. Worth the extra? Eur Heart J. 1991;12(2):218-23.

45. Kehr E, Sono M, Chugh SS, Jerosch-Herold M. Gadoliniumenhanced magnetic resonance imaging for detection and quantification of fibrosis in human myocardium in vitro. Int $\mathrm{J}$ Cardiovasc Imaging. 2008;24(1):61-8. doi:10.1007/s10554-007-9223-y.

46.• Flett AS, Hayward MP, Ashworth MT, Hansen MS, Taylor AM, Elliott PM, et al. Equilibrium contrast cardiovascular magnetic resonance for the measurement of diffuse myocardial fibrosis: preliminary validation in humans. Circulation. 2010;122(2):138 44. doi:10.1161/CIRCULATIONAHA.109.930636. The authors developed an extracellular volume technique and validated it against histology in patients with severe aortic stenosis and hypertrophic cardiomyopathy.

47. White SK, Sado DM, Fontana M, Banypersad SM, Maestrini V, Flett AS, et al. T1 mapping for myocardial extracellular volume measurement by CMR: bolus only versus primed infusion technique. JACC Cardiovase Imaging. 2013. doi:10.1016/j.jemg.2013. 01.011.

48. Miller CA, Naish JH, Bishop P, Coutts G, Clark D, Zhao S, et al. Comprehensive validation of cardiovascular magnetic resonance techniques for the assessment of myocardial extracellular volume. Circ Cardiovasc Imaging. 2013;6(3):373-83. doi:10.1161/ CIRCIMAGING.112.000192.

49. Iles L, Pfluger H, Phrommintikul A, Cherayath J, Aksit P, Gupta $\mathrm{SN}$, et al. Evaluation of diffuse myocardial fibrosis in heart failure with cardiac magnetic resonance contrast-enhanced T1 mapping. J Am Coll Cardiol. 2008;52(19):1574-80. doi:10.1016/j.jacc.2008. 06.049 .

50. Coelho-Filho OR, Shah RV, Mitchell R, Neilan TG, Moreno Jr H, Simonson B, et al. Quantification of cardiomyocyte hypertrophy by cardiac magnetic resonance: implications for early cardiac remodeling. Circulation. 2013;128(11):1225-33. doi:10.1161/ CIRCULATIONAHA.112.000438.

51.• Ugander M, Oki AJ, Hsu LY, Kellman P, Greiser A, Aletras AH, et al. Extracellular volume imaging by magnetic resonance imaging provides insights into overt and sub-clinical myocardial pathology. Eur Heart J. 2012;33(10):1268-78. doi:10.1093/eurheartj/ehr481. The authors show that ECV imaging can quantitatively characterize MI, atypical diffuse fibrosis, and subtle myocardial abnormalities not clinically apparent on LGE images.

52.• Wong TC, Piehler K, Meier CG, Testa SM, Klock AM, Aneizi AA, et al. Association between extracellular matrix expansion quantified by cardiovascular magnetic resonance and short-term mortality. Circulation. 2012;126(10):1206-16. doi:10.1161/ CIRCULATIONAHA.111.089409. In this large cohort study, the authors show in unselected patients that ECV predicts outcomes at least as strongly as left ventricular ejection fraction.

53. Wong TC, Piehler KM, Kang IA, Kadakkal A, Kellman P, Schwartzman DS, et al. Myocardial extracellular volume fraction quantified by cardiovascular magnetic resonance is increased in diabetes and associated with mortality and incident heart failure admission. Eur Heart J. 2013. doi:10.1093/eurheartj/eht193.

54. Piechnik SK, Ferreira VM, Dall'Armellina E, Cochlin LE, Greiser A, Neubauer S, et al. Shortened Modified Look-Locker Inversion recovery (ShMOLLI) for clinical myocardial T1-mapping at 1.5 and $3 \mathrm{~T}$ within a 9 heartbeat breathhold. J Cardiovasc Magn Reson. 2010;12:69. doi:10.1186/1532-429X-12-69. A seminal papers for the three main T1 mapping sequences.

55. Messroghli DR, Radjenovic A, Kozerke S, Higgins DM, Sivananthan MU, Ridgway JP. Modified Look-Locker inversion 
recovery (MOLLI) for high-resolution T1 mapping of the heart. Magn Reson Med. 2004;52(1):141-6. doi:10.1002/mrm.20110. A seminal paper for the three main T1 mapping sequences.

56. Chow K, Flewitt JA, Green JD, Pagano JJ, Friedrich MG, Thompson RB. Saturation recovery single-shot acquisition (SASHA) for myocardial T mapping. Magn Reson Med. 2013. doi:10.1002/mrm.24878. A seminal paper for the three main T1 mapping sequences.

57.• Kellman P, Wilson JR, Xue H, Ugander M, Arai AE. Extracellular volume fraction mapping in the myocardium, part 1: evaluation of an automated method. J Cardiovasc Magn Reson. 2012;14:63. doi:10. 1186/1532-429X-14-63. In this work, the authors developed and evaluated a fully automated, motion-corrected and co-registered method for producing ECV maps feasible for clinical work flow.

58. Sado DM, Flett AS, Banypersad SM, White SK, Maestrini V, Quarta G, et al. Cardiovascular magnetic resonance measurement of myocardial extracellular volume in health and disease. Heart. 2012;98(19):1436-41. doi:10.1136/heartjnl-2012-302346.

59. Karamitsos TD, Piechnik SK, Banypersad SM, Fontana M, Ntusi $\mathrm{NB}$, Ferreira VM, et al. Noncontrast t1 mapping for the diagnosis of cardiac amyloidosis. JACC Cardiovasc Imaging. 2013;6(4):48897. doi:10.1016/j.jcmg.2012.11.013.

60. Sado DM, White SK, Piechnik SK, Banypersad SM, Treibel T, Captur G, et al. The identification and assessment of anderson fabry disease by cardiovascular magnetic resonance non-contrast myocardial T1 mapping. Circ Cardiovasc Imaging. 2013. doi:10.1161/ CIRCIMAGING.112.000070.

61. Bull S, White SK, Piechnik SK, Flett AS, Ferreira VM, Loudon M, et al. Human non-contrast T1 values and correlation with histology in diffuse fibrosis. Heart. 2013;99(13):932-7. doi:10.1136/heartjnl2012-303052.

62. Puntmann VO, Voigt T, Chen Z, Mayr M, Karim R, Rhode K, et al. Native T1 mapping in differentiation of normal myocardium from diffuse disease in hypertrophic and dilated cardiomyopathy. JACC Cardiovasc Imaging. 2013;6(4):475-84. doi:10.1016/j.jemg.2012. 08.019.

63. Ferreira VM, Piechnik SK, Dall'armellina E, Karamitsos TD, Francis JM, Ntusi N, et al. T mapping for the diagnosis of acute myocarditis using CMR: comparison to T-weighted and late gadolinium enhanced imaging. JACC Cardiovasc Imaging. 2013. doi: 10.1016/j.jcmg.2013.03.008.
64.• Moon JC, Messroghli DR, Kellman P, Piechnik SK, Robson MD, Ugander M, et al. Myocardial T1 mapping and extracellular volume quantification: a Society for Cardiovascular Magnetic Resonance (SCMR) and CMR Working Group of the European Society of Cardiology consensus statement. J Cardiovasc Magn Reson. 2013;15(1):92. doi:10.1186/1532-429X-15-92. This document provides recommendations for clinical and research $T 1$ and ECV measurement, addressing site preparation, scan type, scan planning and acquisition, quality control, visualisation and analysis, technical development, as well as discussing controversies in the field.

65. Robson MD, Piechnik SK, Tunnicliffe EM, Neubauer S. T measurements in the human myocardium: the effects of magnetization transfer on the SASHA and MOLLI sequences. Magn Reson Med. 2013. doi:10.1002/mrm.24867.

66. Coelho-Filho OR, Mongeon FP, Mitchell R, Moreno Jr H, Nadruz $\mathrm{Jr} \mathrm{W}$, Kwong R, et al. Role of transcytolemmal water-exchange in magnetic resonance measurements of diffuse myocardial fibrosis in hypertensive heart disease. Circ Cardiovasc Imaging. 2013;6(1): 134-41. doi:10.1161/CIRCIMAGING.112.979815.

67. de Jong S, Zwanenburg JJ, Visser F, der Nagel R, van Rijen $\mathrm{HV}$, Vos MA, et al. Direct detection of myocardial fibrosis by MRI. J Mol Cell Cardiol. 2011;51(6):974-9. doi:10.1016/ j.yjmcc.2011.08.024.

68. Ugander MC, Chen MY, Chen B, Bagi PS, Hsu LY, Kellman P, Arai AE. Contrast Enhanced CT and MRI Measures of Extracellular Volume Fraction Confirm Presence of PeriInfarct Edema in Acute Myocardial Infarction (Abstract). American Heart Association 2011.

69. Bandula S, White SK, Flett AS, Lawrence D, Pugliese F, Ashworth MT, et al. Measurement of myocardial extracellular volume fraction by using equilibrium contrast-enhanced CT: validation against histologic findings. Radiology. 2013. doi: 10.1148/radiol.13130130.

70. Nacif MS, Kawel N, Lee JJ, Chen X, Yao J, Zavodni A, et al. Interstitial myocardial fibrosis assessed as extracellular volume fraction with low-radiation-dose cardiac CT. Radiology. 2012;264(3):876-83. doi:10.1148/radiol.12112458.

71. Butler J, Fonarow GC, Gheorghiade M. Strategies and opportunities for drug development in heart failure. Jama. 2013;309(15): 1593-4. doi:10.1001/jama.2013.1063. 